\title{
Cytological Studies in Some Flowering Dioecious Plants, with Special Reference to the Sex Chromosomes
}

\author{
By \\ Goichi Nakajima \\ Kirihu Girls' High School, Kirihu, Japan
}

Liecitied Alt5ust 7, roft

\section{Materials and Methods}

The present investigation is the continuation of the studies which were previously reported by the same author (1937).

Materials used in this study are as follows:

Salix babylonica L.

S. eriocarpa Franch. et. Sav.

Torreya macrosperma Miyoshi.

Cercidiphyllum japonicum Sieb. et. Zucc.

Picrassima quassioides Benn.

Ilex serrata Thunb. var. Sieboldi Loes.

Actinidia Kolomicta Maxim.

A. polygama Miq.

Eurya japonica Thunb.

Smilax Sieboldi Miq.

Dioscorea japonica Thunb.

These eleven species are entirely dioecious.

Young flower buds were taken from the plants which were growing in the wild. These materials were fixed with Caroncy's mixture excepting Ilex sertuta Thunb. var. Sieboldi Loes. Actinidia Kolomicta Maxim. and Dioscorea joponica Thumb., in which fresh materials were observed in Belling's iron-aceto-carmine. Meiotic chromosomes were observed exclusively in P.M.C. The root tips were fixed with Nawashin's solution from the plants either wild or grown in soiled pots. All the fixed materials were imbedded in paraffin and cut $15-18 u$ thick. The permanent preparations were stained with Heidenhain's iron-alm-haematoxylin.

The figures were drawn by means of Abbe's camera with Zeiss $1,5 \mathrm{~mm}$, Leitz $\frac{1}{12} \mathrm{a}$ and Zeiss compensation ocular $\times \mathbf{1 5}$. Original magnifications are $\times 2030$ for Figs. 14, 15, 21-24 and 27, $\times 2300$ for Figs. 11-13, 25 and $26, \times 2540$ for Figs. 5 and 6 , and $\times 3000$ for Figs. 1-4 and $19-20$.

\section{Results of observations}

1. Salix

Cytological studies in the genus Salix especially in regard to the sex 
chromosomes were first reported by Blackburn and Harrison (1928), and later some reports were published in succession by several investigators, for examples by Blackburn (1929), Sinotô (1929), Håkansson (1933), Nilson (1935), Marklund (1935) and Nakajima (1937). The number of species cytologically studied amounted to 24 or so. The results of these investigations are listed in Table 1.

Table 1. Chromosome numbers and their formula in the genus Salix

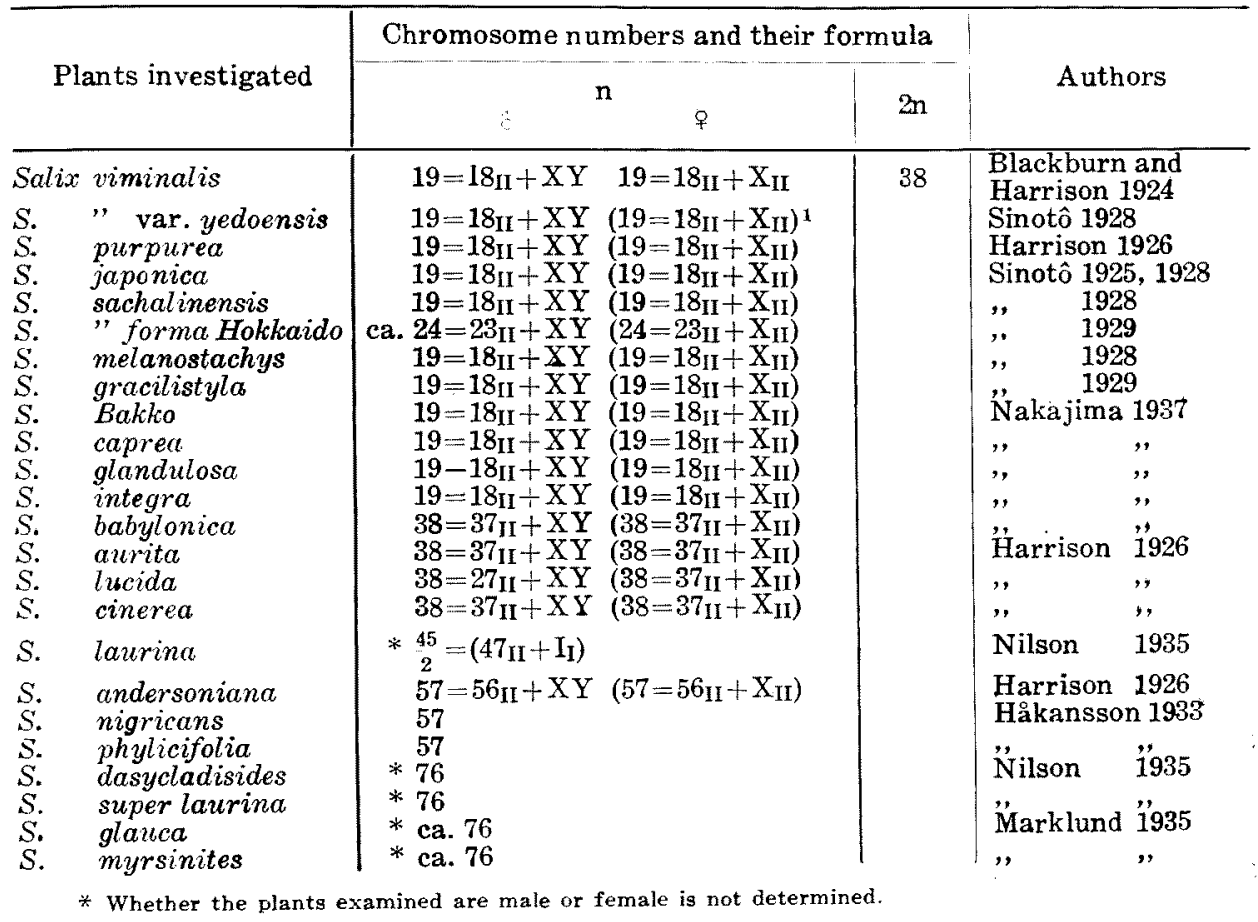

Table 1 shows that there exists a polyploid series in which 19 is the basic number. Besides, the numbers 22 and 24 were observed by Blackburn and Harrison (1924) in some species, for an example in Salix triandra, and ca. 24 were found by Sinotô (1929) in S. sacharinensis f. Hokkaido. In male individuals of the above cited polyploid species, it was observed by investigators that there are one unequal pair of chromosomes consisting of a large and a small member.

In the present investigation, I have studied two species, Salix babylonica and S. eriocarpa, both of which being well known as weeping willows and are found quite frequently in every parts of Japan.

Salix babylonica. should be entirely dioecious, but I could not find any

1 The numbers of chromosomes and their formulae in the parentheses in this table were not examined, but assumed to be so. 
female individuals in ca. one hundred individuals examined. All of the plants were male as the same as in Dioscorea Batatas and Helwingia japonica, in each of which I could observe none of the female individual.

In male individuals of $S$. babylonica and $S$. eriocarpa, I observed 38 gemini in heterotypic metaphase, so that these species are of tetra-basic (Figs. 1,3). The chromosomes are small and spheroidal in shape and are considerably different in size. In side views of the heterotypic metaphase of these 2 species, I could clearly observe one unequal pair of chromosomes (Figs. 2, 4). Thus the sex chromosomes are of XY-type.

\section{Torreya macrosperma Miyoshi}

The cytological investigations in Taxaceae which includes the genus Torreya were made by Darlington (1930), Dark (1932), Sax (1933) and Matsuura and Sutô (1935), etc. in 4 species of Taxas, namely in $\mathrm{T}$. baccata, $T$. canadensis, $T$. cuspidata and $T$ Hunnewelliana. The reduced chromosome numbers were reported by these authors to be 12 in 3 species excluding $T$. cuspidata in which 12 and 13 were respectively counted in male and female individuals.

The reports of the cytological study on the genus Torreya are now quite scarce. In Torreya macrosperma, that was used in the present investigation, it was difficult to fix the flower buds as the resin is contained in the buds. The fixation was done in the earlier spring time.

In Torreya macrosperma, 11 bivalents were observed in polar views of heterotypic metaphase in male plants. The gemini are similar in size and shape (Fig. 5). In side views of the heterotypic metaphase, the present writer observed one unequal pair of chromosomes consisting of the two members of different size (Fig. 6). Thus the sex chromosomes are of XY-type.

3. Cercidiphyllum japonicum Sieb. et. Zucc.

Whitaker (1933) has reported that the reduced number of this species is 19 . I counted also 19 chromosomes in polar views of heteroand homotypic metaphases of male plant in the same species (Fig. 8). The chromosomes are varying in size and shape. In side views of the heterotypic metaphase, I observed one unequal pair consisting of two elements of different size, one of them being about twice as large as the other as showing in Fig. 7. The two members of this pair were so different in size that I could easily recognize them in heterotypic anaphase on the way passing to different poles. The sex chromosomes are of XY-type.

4. Picrassima quassioides Benn.

This plant is commonly known as a medicinal plant, its bark being 


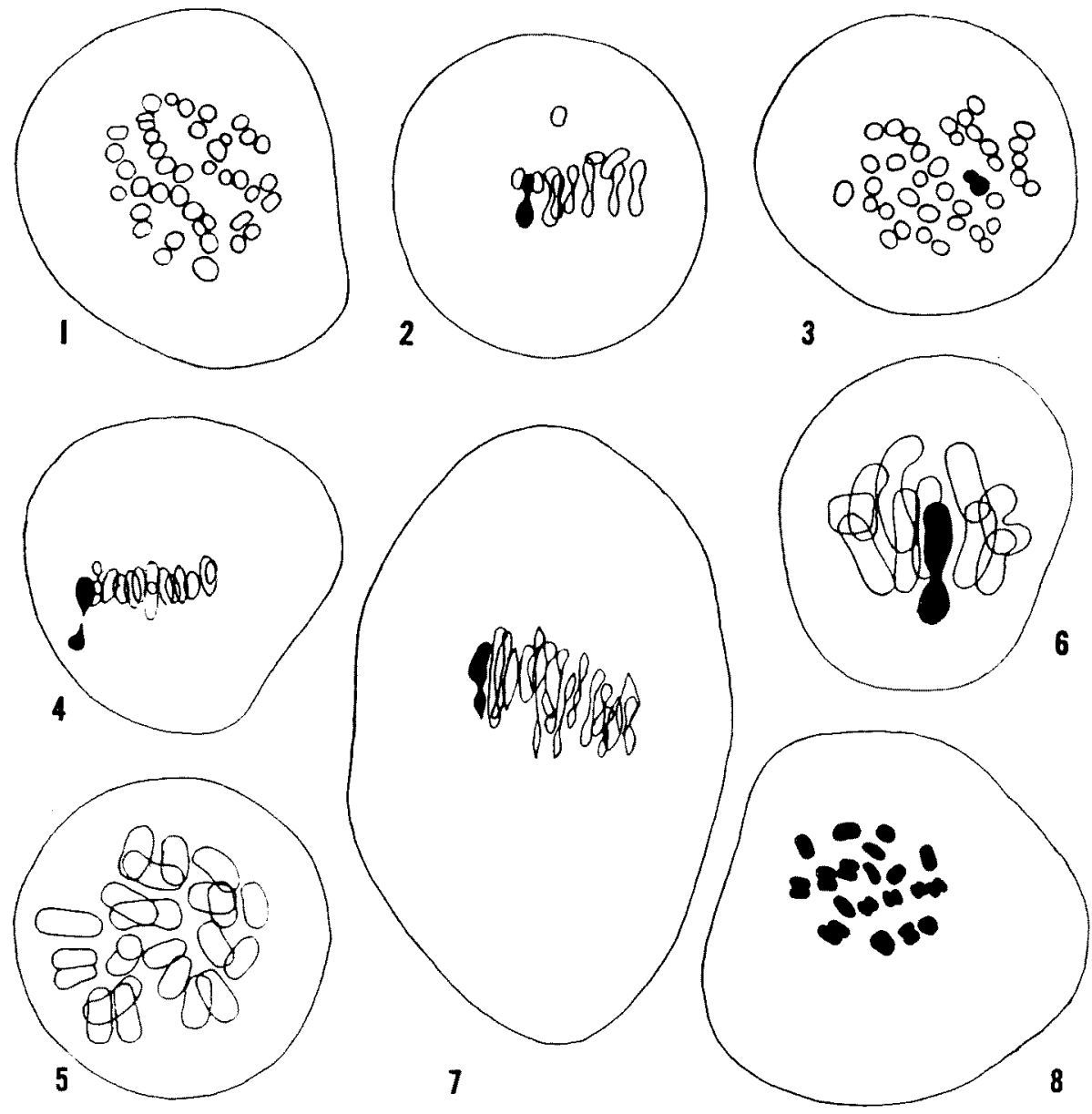

Figs. 1-8. 1-2. Salix babylonica. $\times 2000.1$, polar view of heterotypic metaphase showing 38 gemini. 2, side views of the same stage; unequal pair in black. 3-4. Salix eriocarpa. $\times 2000$. 3, polar view of heterotypic metaphase showing 38 gemini ; unequal pair in black. 4, side view of the same stage; unequal pair in black. 5-6. Torreya macrosperma. $\times 1700$. 5 , polar view of heterotypic metaphase showing 11 gemini. 6, side view of the same stage; unequal pair in black. 7-8. Cercidiphyllum japonicum: $\times 2000.7$, side view of heterotypic metaphase; unequal pair in black. 8, chromosome group in homotypic metaphase, showing 19 univalents.

used as a stomatic and an anthelmintic. In male plants, the reduced numbers were counted to be 25 at metaphase in hetero- and homotypic divisions (Fig. 9). The chromosomes do not differ much in their size, but fourteen elements appear to be more or less larger than other nine. Although I could not find heterogemini in polar views of heterotypic metaphase, one unequal pair were clearly observed in side views of the same stage as shown in Fig. 10. The sex chromosomes are of XY-type. 
5. Ilex serrata Thunb. var. Sieboldi Loes.

According to Sugiura $(1931,1936 \mathrm{~b})$, the somatic number of chromosomes of Ilex crenata is 34 , thus 17 is the reduced number. The present

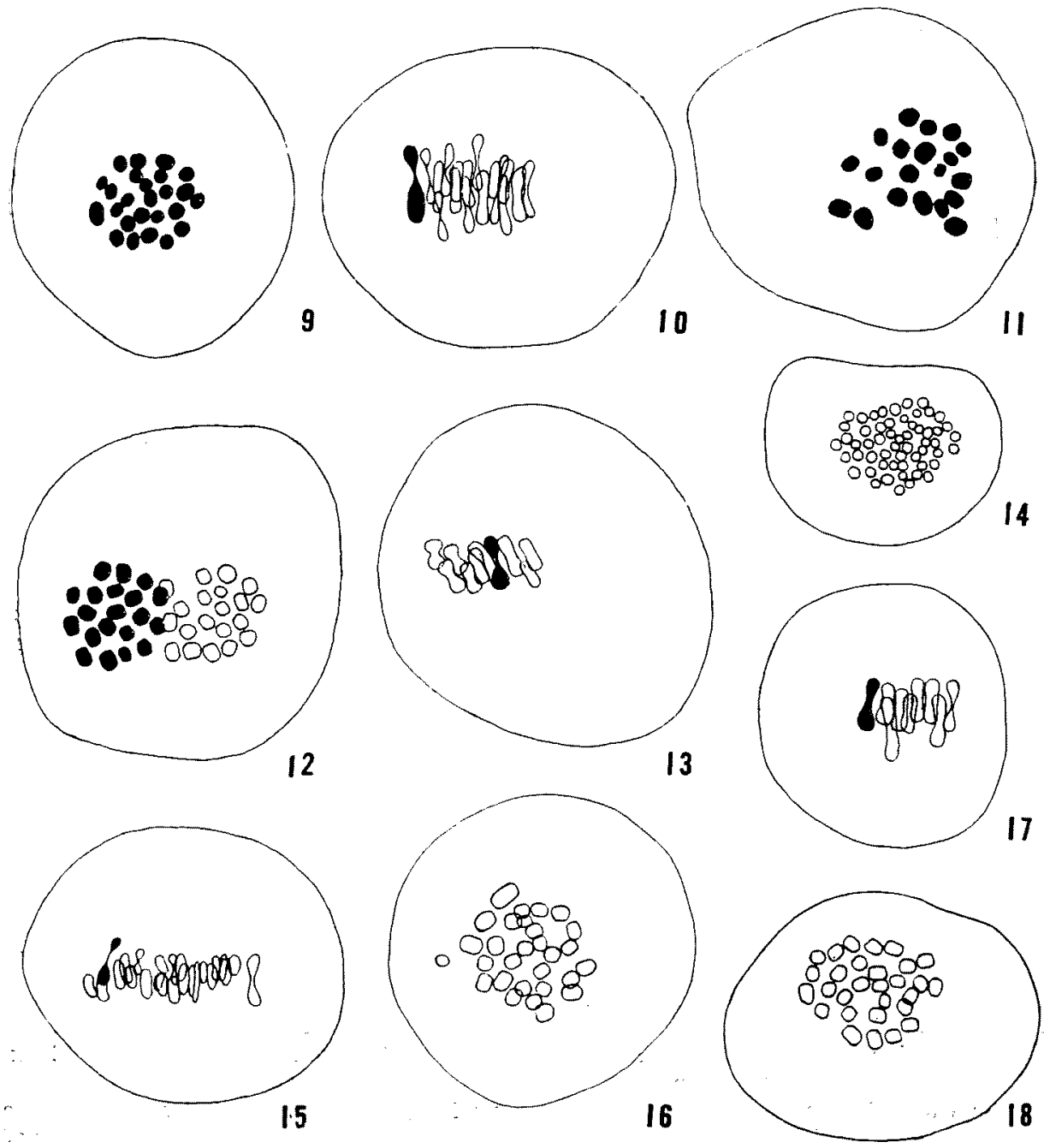

Figs. 9-18. 9-10 Picrassima quassioides. $\times 2000.9$, polar view of heterotypic metaphase showing 25 chromosomes. 10, side view of the same stage; unequal pair in black. 11-13. Ilexs errata var. Sieboldi. $\times 1500$. 11, polar view of heterotypic metaphase showing 20 gemini. 12, two chromosome groups in heterotypic anaphase. 13, side view of heterotypic metaphase; unequal pair in black. 14-15. Actinidia Kolomicta. $\times 1400$. 14, polar view of homotypic metaphase showing 56 chromosomes. 15, side view of heterotypic metaphase; unequal pair in black. 16-18, Actinidia polygama. $\times 1900,16$, polar view of heterotypic metaphase showing 29 gemini. 17, side view of the same stage; unequal pair in black. 18 , polar view of homotypic metaphase, showing 29 chromosomes.

writer observed 20 reduced chromosomes in male plants of $I$. serrata var. Sieboldi at polar views of hetero- and homotypic metaphases (Figs. 11, 
12). They are spheroidal and varying in their size. One of the gemini is especially small.

Although I could not detect any heteromorphic pair in polar views of heterotypic metaphase in male plants of this variety, one unequal pair more or less differing in the size of its elements, was recognized in side views of the same stage as shown in Fig. 13. The sex chromosomes are of XY-type.

6. Actinidia Kolomicta Maxim, and A. polygama Miq.

In Dilleniaceae including the genera Actinidia, Wormia and so forth, only Wormia suffruticosa was studied cytologically by Paetow (1931), and the reduced number was reported to be 13 . The present writer studied two species of the genus Actinidia, i.e. A. Kolomicta and $A$. polygama.

In $A$. Kolomicta the reduced number was found to be ca. 56 at polar views in homotypic metaphase of male plants (Fig. 14). The chromosomes are very small and unequal in size.

In male plants of $A$. polygama I observed ca. 29 reduced elements at polar views of hetero- and homotypic metaphases. The chromosomes are varying in size and about twice as large as those of A. Kolomicta (Figs. $16,18)$.

In male plants of these two species, the chromosomes were so small that I could not detect unequal pairs but in side views of the same stage, I could observe one heteromorphic pair consisting of a large and a small elements as shown in Figs. 15 and 17. The sex chromosomes are of XY-type.

7. Eurreya japonica Thunb.

Eurreya belongs to Theaceae. Some species of Thea, for examples Thea sinensis, T. assamica and $T$ macrophylla were studied cytologically by Simura (1935), Karasawa (1935) and Yamashita (1937), etc., and they reported 15 and 30 as the reduced number in different species that were examined. Further, the presence of triple form was reported besides the diploid and tetraploid forms. But, cytological studies in the genus Eurreya are now quite scarce.

In male plants of Eurreya japonica I observed 21 gemini in the heterotypic metaphase. One of the bivalents was found to be especially large, other gemini also showing variation in size (Fig. 19). Although, I could not detect any unequal pair in polar views of heterotypic metaphase in male plants, one unequal pair was observed in side views of the same stage as shown in Fig. 20. The sex chromosomes are of XY-type.

\section{Smilax Sieboldi Miq.}

Cytological studies in the genus Smilax were reported by Humphrey 
(1914), Elkins (1914), Lindsay (1929, 1930), Jensen (1937), Nakajima $(1933,1937)$ and others. These results are shown in Table 2.

Table 2. Chromosome numbers and their formula in the genus Smilax

\begin{tabular}{|c|c|c|c|}
\hline \multirow{2}{*}{ Plants investigated } & \multicolumn{2}{|c|}{ Chromosome numbers and their formula } & \multirow{2}{*}{ Authors } \\
\hline & $\mathrm{n}$ & $2 \mathbf{n}$ & \\
\hline $\begin{array}{ll}\text { Smilax } & \text { herbacea } \\
\text { S. } & \text { " var.nipponica } \\
\text { S. } & \text { Oldhami } \\
\text { S. } & \text { "forma } \\
\text { S. } & \text { "china } \\
\text { S. } & \text { Sieboldi } \\
\text { S. } & \text { glauca } \\
\text { S. } & \text { lotundifolia }\end{array}$ & $\begin{array}{c}12 \\
13 \\
15=14_{I I}+X Y \\
16=15_{I I}+X Y \\
16+1 f=15_{I I}+X Y+1 f \\
30=29_{I I}+X Y \\
14 \\
16\end{array}$ & 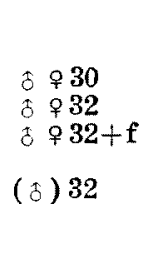 & $\begin{array}{ll}\text { Humphrey } & 1914 \\
\text { Elking } & 1914 \\
\text { Nakiflma } & 1937 \\
\text { ", } & \text { " } \\
\text { " } & \text { "' } \\
\text { Jeneen } & 1933 \\
\text { ", } & \text { ", }\end{array}$ \\
\hline
\end{tabular}

As is shown in Table 2, the reduced numbers are reported to be 12 , $13,14,15,16$ and 30 in this genus. The existence of sex chromosomes
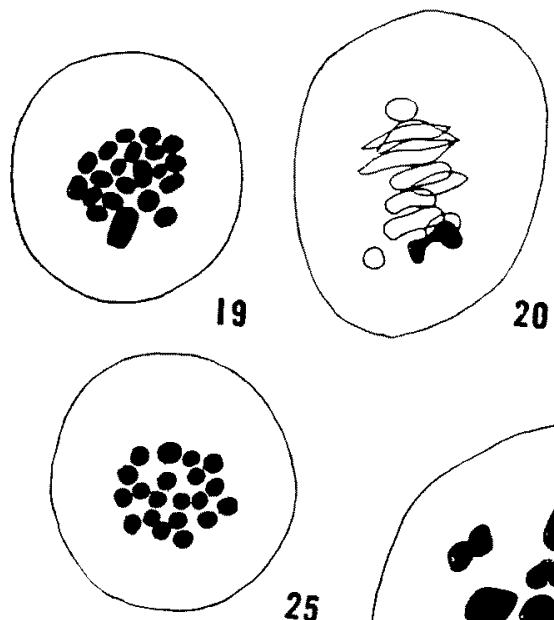

25

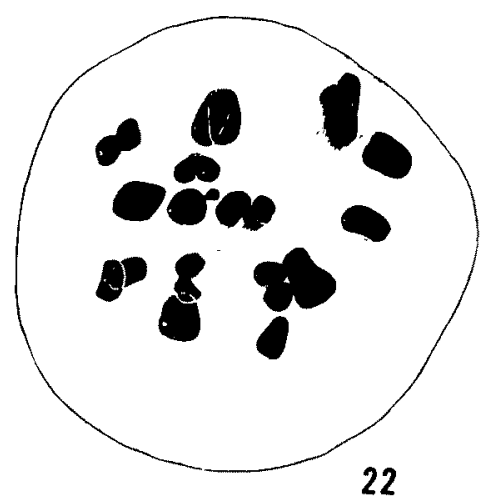

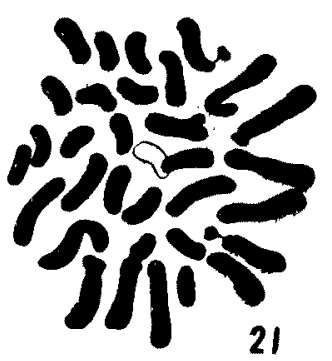

21
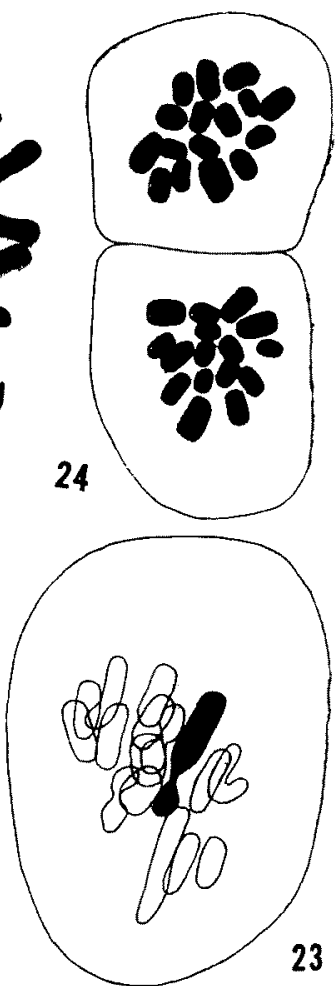

Figs. 19-26. 19-20, Eurreya japonica. $\times 2000 . \quad 19$, polar view of homotypic metaphase showing 21 chromosomes. 20, side view of heterotypic metaphase; unequal pair in black. 21-24. Smilax Sieboldi. $\times 1400.21,32$ somatic chromosomes in a male plant. 22, polar view of heterotypic metaphase showing 16 gemini. 23, side view of the same stage; unequal pair in black. 24, polar view of 2 plates in homotypic metaphase, showing 16 chromosomes. 25-26. Dios:orea japonica, $\times 2000.25$, polar view of heterotypic metaphase showing 20 gemini. 26, side view of the same stage ; unequal pair in black. 
(XY-type) was reported only by Nakajima (1937) in three species and one variety studied by him, though such was not recognized in other species studied by others.

In his previous paper, the present writer reported 32 as the number of somatic chromosomes of some individuals in Smilax Sieboldi, the sex of which was unknown. The same writer found in the present study the same number (Fig. 21) in male individuals. These somatic chromosomes are rod shaped or curved and their sizes are various. Two of the large members are provided with a satellite in each.

In polar views of the hetero- and homotypic metaphases in male plants of this species 16 chromosomes corresponding to the somatic number were counted (Figs. 23, 24). Although the unequal pair could not be found in polar views of heterotypic metaphase, one such as observed in side views of the same stage as shown in Fig. 23. The sex chromosomes are of XY-type.

\section{Dioscorea japonica Thunb.}

The cytological investigation in the genus Dioscorea has been made by Meurman (1925) for the first time. Later the same genus were studied by Nakajima (1933, 1936, 1937), Jensen (1937), Smith (1937) and others. These results are shown in Table 3.

Table 3. Chromosome numbers in the genus Dioscorea

\begin{tabular}{|c|c|c|c|c|}
\hline \multirow{2}{*}{ Plants investigated } & \multicolumn{2}{|c|}{ Chromosome numbers } & 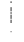 & \multirow{2}{*}{ Authors } \\
\hline & $\mathrm{n}$ & $2 n$ & & \\
\hline $\begin{array}{ll}\text { Dioscorea caucasca } \\
D . & \text { Tokoro Makino } \\
D . & \text { gracillima Miq. } \\
D . & \text { quinquiloba } \\
D . & \text { sinuata } \\
D . & \text { japonica } \\
D . & \text { discolor } \\
D . & \text { maeroura } \\
D . & \text { sp. } \\
D . & \text { quaternata } \\
D . \text { sativa }, \text { L. f. spontanea, Makino } \\
D . & \text { villosa } \\
D . & \text { reticulata } \\
D . & \text { Forgesii } \\
D . & \text { bulbifera } \\
D . & \text { alata } \\
D . & \text { Batatas, Decne } \\
D . & \text { Batatas } \\
D . & \text { Cayenensis } \\
D . & \text { oppasilifolia } \\
D . & \text { pentaphylla }\end{array}$ & 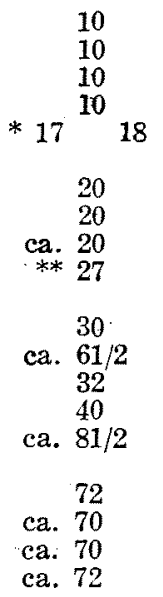 & $\begin{array}{l}20 \\
20 \\
\\
40\end{array}$ & i & 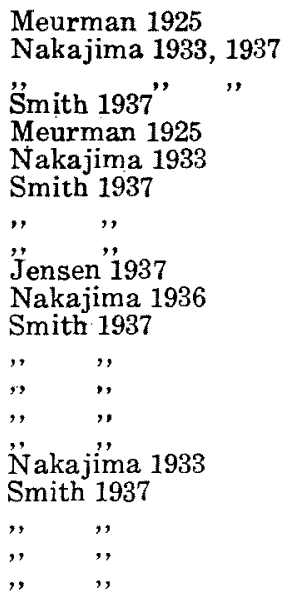 \\
\hline
\end{tabular}

As shown in Table 3, almost all the species are included in a polyploid series having 10 as the basic number. The numbers reported for 
several species which deviate from the polyploid number might have come from the observers' mistake, as the chromosomes are not only quite small but also irregularily divided in meiosis in some species just like in $D$. Batatas. On the other hand, the existence of some aneuploid may be expected also.

The number of somatic chromosomes of $D$. japonica was previously reported by the present writer (1933) to be 40 in male individuals. In the present study 20 gemini, corresponding to the somatic number were observed at polar views of hetero- and homotypic metaphases in male plants (Fig. 25). The bivalents are almost spherical and scarcely vary in size. Although the unequal pair was not detected in polar views of heterotypic metaphase, I could observe very clearly in side views of the same stage one such pair consisting of two elements of different size, one being about five or six times as large as the other as shown in Fig. 26. The sex chromosomes are of XY-type.

\section{Literature cited}

1) Blackburn, K. B. (1929) On the occurrence of sex chromosomes in flowering plants with some suggestion as to their origin. Proc. of the Internat. Congr. of Plant Sci. 1.

2) - and Harrison, J. W. H. (1922) The meiotic phase in the Salicaceae. Rept. Brit. Ass. for the Adv. Sci., (cited from 1).

3) - and - (1924) A preliminary account of the chromosomes and chromosome behaviour in the Salicaceae. Ann. Bot. 38.

4) Elkins, M. G. (1914) The maturation phase in Smilax herbacea. Bot. Gaz. 57. (cited from 8).

5) Harrison, J: W. H. (1926 a) Heterochromosomes and polyploides. Nature 11 (cited from 1 ).

6) - (1929 b) Polyploidy and sex chromosomes. ib. (cited from 1).

7) Humphrey, L. E. (1914) A cytological study of the stamens of Smilax herbacea. Obio Nat. 15. (cited from 12).

8) Lindsay, R. H. (1930) The chromosomes of some dioecious Angiosperms. Amer. Journ. Bot. 17.

9) Meurman, O. (1925) The chromosome behaviour of some dioecious plants and the relation with special reference to the sex chromosomes. Soc. Sci. Fenn. Comm. Biol. 2.

10) Nakajima, G. (1933) Chromosome numbers in some Angiosperms. Japan. Journ. Gen. 9.

11) - (1937) Cytological studies in some dioecious plants. Cytologia, Fujii Jubilee Vol.

12) Sinotô, Y. (1929) Chromosome studies in some dibecious plants, with special reference to the allosome. Cytologia, 1.

13) Sugiura, T. (1931) A list of chromosome numbers in Angiospermous plants. Bot. Mag. (Tokyo) 45.

14) - (1936 b) Studies on the chromosome numbers in higher plants, with special reference of cy tokinesis I. Cytologia 7 .

15) Tischler, G. (1938) Pfanzliche Chromosomen-Zahlen 4. Tab. Biolog. 16. 